\title{
Analysis of the proteomic profiling of brain tissue in Alzheimer's disease
}

\author{
T. Tsuji and S. Shimohama* \\ Department of Neurology, Faculty of Medicine, Kyoto \\ University, 54 Shogoin-Kawaharacho, Sakyoku, Kyoto \\ 606, Japan
}

In proteome analysis, it is necessary to separate proteins as a first step prior to characterization. Thus, the overall performance of the analysis depends strongly on the separation tool, which is usually two-dimensional electrophoresis (2DE). We have utilized 2DE to begin characterization of the complex pathologic processes in Alzheimer's disease (AD). In the present study, we show how a reliable 2-DE database of brain proteins in Alzheimer's disease was created, improving reproducibility by using an immobilized $\mathrm{pH}$ gradient (IPG) for the first dimension gel electrophoresis. The recent progress in this field, and future prospects in this area are also discussed. Preparation of brain proteins into a suitable solubilized state enabled us to separate over 1000 well-defined protein spots in each 2-DE. A comparison of the density of the spots identified on the reference map between the $\mathrm{AD}$ and control group, showed that 5 protein spots were significantly increased, 28 spots were significantly decreased and 7 spots were specifically detected in AD. Two spots among those significantly increased and one spot among those significantly decreased were identified as GFAP related. It is hoped that comparative studies to identify, quantitate, and characterize the proteins differentially expressed in normal brain versus diseased brain will give insight into the mechanisms of pathogenesis and allow the development of a strategy to control both the etiology and course of the diseases.

Keywords: Two-dimensional gel electrophoresis, Alzheimer's disease, database, Internet

\footnotetext{
*Address for correspondence: S. Shimohama, MD, PhD, Department of Neurology, Faculty of Medicine, Kyoto University, 54 Shogoin-Kawaharacho, Sakyoku, Kyoto 606, Japan. Tel.: +81 75 751 3767; Fax: +81 75751 9541; E-mail: i53367@ sakura.kudpc. kyoto-u.ac.jp.
}

\section{Introduction}

\subsection{Alzheimer's disease}

Neural cells are unique in as much as they are unable to multiply after birth and throughout an individual's lifetime. The reason for such longevity is thought to be the good post-translational control of proteins. The most important pathological feature of the brain in aging and Alzheimer's disease (AD) is the presence of aggregated $\beta$-amyloid protein and neurofibrillary tangles (NFT), which are much more prominent in AD brains compared with age-matched non-AD brains. These pathological features have already been described in the original article written by Alzheimer himself. Since then, nearly one century, the precise characterization of these materials has caught the attention of many researchers, but the mechanism of accumulation of these proteins remains unknown. Clarification of the precise mechanism of protein metabolism is an important goal which is expected to be achieved during this century.

Recent research on AD has yielded many fruitful and rapidly unfolding observations relating to its pathogenesis. Various proteins such as amyloid precursor protein (APP), $\beta$-amyloid, tau, presenilin and apoE are likely to be involved in the development of this disease [1-7]. Recent advances in molecular biology techniques have enabled us to identify the possible candidate genes for familial AD [8-11]. These mutations have been thoroughly analyzed in relation to the pathological process. Studies at the protein level, however, have lagged partly due to the complexity of the techniques required for protein separation, analysis, and identification.

We are developing amazing tools to enable us to investigate whole sets of translated proteins [12], called proteomes (protein complement expressed by the genome in a particular cell or tissue). This will make it possible to map the relationship of whole proteins and their changes in aging and neurodegenerative diseases, such as AD. 


\subsection{Proteome analysis}

During the last few years, considerable effort has focused on genomic studies and large-scale DNAsequencing projects. The ultimate goal of these studies is to define the genome, the human genome in particular, with the hope of being able to identify and study key genes functional in both normal and aberrant pathways in diseases. Several genetic alterations must be considered in the development of neurodegenerative diseases such as AD, especially in cases with rapid progression. It has been estimated, however, that only $2 \%$ of human diseases result from single gene defects (i.e. expression of an altered gene or loss of function of a normal gene). Epigenetic and environmental factors are involved in the remaining $98 \%$ of human diseases [13]. While gene expression has traditionally been studied at both the messenger RNA (m RNA) level and the protein expression level, it should be emphasized that mRNA-based studies only identify message abundance and not the actual levels of proteins, which are the functional molecules within the cell wall. In fact, Anderson and Seihamer have recently shown that, in the human liver, there may not be a good correlation between mRNA abundance and the amount of protein present [14]. Analysis of protein expression may provide a better assessment of the metabolic state of cells.

Protein analysis, however, is considerably more challenging than DNA/RNA-based analyses, since, in contrast to DNA, which is composed of only four different nucleotides, proteins are composed of at least 20 unmodified, and many more modified, amino acids; thus the physicochemical characteristics of proteins vary considerably. Adding to the complexity of analysis is the fact that many proteins undergo innumerable co- and posttranslational modifications, including phosphorylation, acetylation, sialation, glycosylation, myristoylation, conjugation with lipids, and proteolytic processing.

In contrast to the genome, which represents the fixed digitized characteristics of an organism, the proteome is expressed as a constant state of protein metabolism and is dependent upon multiple factors, including the developmental state of the organism, tissue and organelle location, and metabolic stage. Comparative studies to identify, quantitate, and characterize the proteins expressed in normal versus diseased cells will give insight into the mechanisms of pathogenesis.

\section{Methodology of proteome analysis}

\section{1. $2 D-P A G E$}

Two-dimensional gel electrophoresis (2-DE) has been developed as a method of protein separation combining isoelectric focusing gel electrophoresis (IEF) with sodium dodecyl sulfate (SDS) polyacrylamide gel electrophoresis (PAGE). This combination can separate and characterize many thousands of proteins detected as spots on the gels or transferred membranes [15]. The measurement of changes in the expression of multiple proteins represents a powerful strategy for characterizing complex pathophysiologic processes and designing novel drug therapies. Numerous technological improvements and modifications have been made to the original 2D-PAGE protocol. Approaches have focused on the development of reproducible and standardized procedures for sample preparation and the running of gels, enhanced spot detection and accurate protein identification, development of computer software for the analysis of polypeptide spot patterns and database construction.

\subsection{Reproducibility and standardization of spot patterns}

Recently, immobilized $\mathrm{pH}$ gradient (IPG) electrophoresis has been introduced to eliminate complications associated with the use of carrier ampholytes (Cas) in the first dimension IEF in 2D-PAGE. IPG gel strips (Pharmacia), formulated in a variety of narrow $(\mathrm{pH}<1)$ and broad $(\mathrm{pH} 3-10)$ range gradients, offer many advantages not afforded by CA-IEF including (i) enhanced gel pattern reproducibility, thereby greatly facilitating inter- and intralaboratory gel pattern comparisons; (ii) increased spot resolution and detection sensitivity over wide $\mathrm{pH}$ ranges; (iii) enhanced loading capacity for subsequent micropreparative applications. Whereas CA-IEF is essentially limited to the separation of microgram quantities of proteins between $\mathrm{pH} 4.5-$ 7.5, IPG-2D-PAGE permits the separation of milligram (1-15 mg) quantities of proteins on a single IP strip [16, 17]. In addition, IPG strips are considerably easier to manipulate than tube gels, since the IPG gels are covalently bound to mylar backing strips and are not subject to gel stretching and breakage, minimizing geometric distortions in gel patterns accordingly. Intraor inter- laboratory studies comparing the reproducibility of 2D-PAGE patterns using IPG gels in the first dimension showed a less than 1-mm positional variability between spots observed in standardized protein samples [18-21]. 


\subsection{Enhanced spot detection/sensitivity}

Since most physiological and pathological processes are associated with a quantitative modulation of gene expression, sensitive spot detection and accurate protein quantitation methodologies are of utmost importance. The use of broad range ( $\mathrm{pH} 4-12)$ IPGs provides an excellent global overview of protein composition. Separations utilizing well-defined, narrow range (1-2 pH unit) IPGs, specifically designed for the proteins of interest, have permitted the detection and analysis of individual polypeptides and polypeptide isoforms (e.g. phosphorylation, glycoproteins, etc.) having pIs differing by as little as $0.01 \mathrm{pH}$ units. While the lower limit of spot detection using current silver staining methods is approximately $1 \mathrm{ng}$ [22] or roughly 10 copies per cell [18], immunoblotting, using highaffinity monoclonal antibodies and visualization with enhanced chemiluminescence can detect as few as 110 copies per cell. Microheterogeneity of m-calpain in the cytosolic fraction and possible changes in $\mathrm{AD}$ were detected with high-resolution 2-DE [23]. In the detection of the m-calpain molecule, quantitative information was gained as well as identification of various isoforms that most likely result from posttranslational modification of the primary gene products represented by eight spots on the transferred membranes. Although no qualitative changes in the microheterogeneity were found in $\mathrm{AD}$, (all the spots were detected in both AD and controls), calculation of the ratio of the four acidic spots to the total calpain spots on silver stained 2-DE gels showed significant increase in the ratio of acidic spots in $\mathrm{AD}$.

\subsection{Computer analysis/database development/www access}

Although 2D-PAGE maps appear complex, the technique is highly reproducible, and simple analysis of the 2D-PAGE patterns can be performed by superimposing one photographic image over another on a light box. For more complex studies, sophisticated computer analysis software packages have been developed to aid in the scanning and digitalization of 2D-PAGE maps, quantitation of individual protein spots and the automatic matching of gel images [24-28]. The high reproducibility of IPG-2D-PAGE has permitted the generation of several high-resolution 2D-PAGE reference maps and comprehensive databases of qualitative and quantitative protein expression in a variety of cell types and tissues [29].

\section{Brain proteome analysis in Alzheimer's disease}

Several laboratories are constructing 2-DE databases and providing them via the Internet to researchers world wide (http://www.expasy.ch; http://biobase.dk/cgi-bin/ celis; http://www-ips.ncifcrf.gov/lemkin/gellab.html; http://siva.cshl.org/index.html). Using such databases, 2-DE data in individual laboratories can be compared with normal standards in order to detect changes in spots. Although the specimens used to construct these databases have become more diversified, they are currently restricted mainly to cultured cells, blood components, cardiac muscle, and liver proteins. There is not a 2-DE database for human brain proteins presently available on the Internet, which may reflect the heterogeneity of brain tissues that could produce inconsistent 2-DE images as well as the relatively low reproducibility of 2-DE separation of brain proteins.

The most important step to permit 2-DE analysis in $\mathrm{AD}$ is to establish a reference map for constructing 2-DE databases. In the present study, we established a reference map of human brain proteins and demonstrated specific changes in protein spots in AD by improved reproducibility of the 2-DE analysis. Compared with classical 2-DE using CA, 2-DE electrophoresis using IPG, which are integral parts of the polyacrylamide matrix, has produced significant improvements in 2-DE electrophoretic separation, permitting higher resolution and reproducibility [20,30-32]. Using IPG for the first dimension of electrophoresis, we could use SDS as a protein solubilization reagent for IEF [33]. We constructed a reference map with Melanie II software (Bio-Rad Inc. Richmond, CA) by collecting wellmatched spots within selected gels in order to reduce errors related to the process of making a reference gel. With these improvements, we could analyze protein changes in disease after constructing a 2-DE map of human brain proteins. In the present study, we sought to establish a 2-DE database by identifying several protein spots on the 2-DE map in control specimens and applied the database to the detection of specific changes in these spots in AD.

\section{Materials and methods}

\subsection{Autopsy brain samples}

Brain tissues were obtained at autopsy from 15 patients diagnosed clinically and histopathologically with $\mathrm{AD}$ (63 to $94 \mathrm{yr}$, postmortem period 4 to $21 \mathrm{hr}$ ), and 
from 15 age-matched controls ( 60 to $87 \mathrm{yr}$, postmortem period 4 to $24 \mathrm{hr}$ ) with no clinical or morphologic evidence of brain pathology. In both groups the typical cause of death was cardiac failure or a terminal respiratory condition. Immediately after autopsy the brains were divided saggitally into halves with one half used for biochemical studies and the other half for histological examination. The temporal cortices were used in the present study. The influence of the postmortem period on the 2-DE pattern was examined in three groups with a different postmortem period; early (4-8 hr), middle (9-16 hr) and late (17-24 hr).

\subsection{Reagents}

IPG gradient gel strips ( $\mathrm{pH} 4.0-7.0)$ and RepelSilane were purchased from Pharmacia IPG (Bromma, Sweden). SDS and 2-D markers were obtained from Bio-Rad. Phenylmethylsulfonyl fluoride (PMSF) and iodoacetamide were obtained from Sigma Chemicals (St Louis, MO). Monoclonal anti- $\beta$ actin antibody (clone no. AC-15) and an anti-glial fibrillary acidic protein (GFAP) antibody (clone no. G-A-5) were purchased from Sigma Chemicals. All other chemicals were obtained from Nakarai (Kyoto, Japan).

\subsection{Sample preparation}

Brain tissues were thoroughly sonicated with a hand sonicator in $1 \mathrm{v} / \mathrm{w}$ of lysis buffer which contained $10 \mathrm{mM}$ Tris $\mathrm{HCl}(\mathrm{pH} 7.5), 2 \%$ SDS, and 2\% mercaptoethanol. After centrifugation at $100000 \mathrm{~g}$ for $1 \mathrm{hr}$, the supernatant was collected and diluted with sample buffer containing $9 \mathrm{M}$ urea, $0.5 \%$ Triton-X 100, and $0.14 \%$ PMSF.

\subsection{The 2-DE system}

The first dimension of gel electrophoresis was carried out using an immobilized $\mathrm{pH}$ gradient gel (immobilized dry strip gel, $\mathrm{pH} 4-7 / 18 \mathrm{~cm}$, Pharmacia) with a horizontal electrophoresis apparatus (Multiphor II, Pharmacia) according to the method described by Gorg et al. [34]. The sample solution was applied on the anodic side of the gel and was run according to manufacturer's instructions. The second dimension of gel electrophoresis was carried out on a $15 \%$ running gel $(20 \mathrm{~cm} \times 20 \mathrm{~cm} \times 0.1 \mathrm{~cm})$ in the presence of SDS essentially as described by Laemmli [35]. When necessary, marker proteins (SDS-PAGE standards and 2D standards from Bio-Rad) were separated in the same way to estimate the isoelectric points and molecular weights.

\subsection{Protein staining}

After gel electrophoresis in the second dimension, the protein spots were visualized by silver staining with a Wako silver stain kit II (Wako Pure Chem. Ins., Osaka, Japan) which can detect $15 \mathrm{ng}$ protein on 2-D separated spots.

\subsection{Immunoblotting}

Immunodetection is a powerful and sensitive technique which relies on the specificity of antibodies to identify single protein spots from 2-D PAGE. Immunoblotting using commercially available antibodies and the enhanced chemiluminescence (ECL) system (Amerciam, UK) was carried out to identify $\beta$-actin and GFAP on the 2-DE reference map to establish landmarks to facilitate comparison with other 2-DE reference maps.

\subsection{Data analysis}

Protein spots on silver-stained 2-DE gels were digitized using a flatbed scanner at 300 dpi (Agfa-Gevaert, Mortsel, Belgium). The image data were analyzed on a Macintosh computer (Power Macintosh 7600/132) with Melanie II software (Bio-Rad). We analyzed the spots without filtering images to avoid artificial effects on images in comparisons. Spots detected by the program were matched between each gel in each group, and a reference gel was produced by merging the spots from the gels studied. When all gels had been matched to the given reference gel, the latter provided a unique numbering scheme for spot features across all gels. Each paired spot feature in a gel image could then be compared with the corresponding feature in the reference gel. The spot features were characterized with respect to optical density, area and volume. The volume was calculated by integration of the optical density (OD) over the spot's area. Relative volume (\% VOL) was the ratio of VOL to total VOL over the whole image. The $\%$ VOL of the spots was analyzed to detect specific spots representing significant differences between $\mathrm{AD}$ and control groups. Data were analyzed statistically with Statview IV on a Macintosh, enabling us to identify spots in AD significantly changed from the controls by one-way analysis of variance and Bonferroni/Dunne's $\mathrm{t}$ test, defining siginificance as $p<0.05$. 


\section{Results}

\subsection{Detection of protein spots on the gels}

A photograph of a 2-D gel, representing the unfiltered raw image demonstrates high-resolution separation of spots and low background staining (Fig. 1). About 700 spots were assigned to a synthetic reference gel, including well-matched spots shared with the control group. Protein spots heavier than $100 \mathrm{kDa}$ were far fewer than proteins lighter than $100 \mathrm{kDa}$. No significant difference was noted between the characteristics of the spots in the samples from the different postmortem periods (data not shown). Two individual protein spots were respectively assigned to $\beta$-actin and GFAP by immunoblot analysis.

\subsection{Protein spots lost or significantly decreased in AD brain}

There were no protein spots present in the controls but absent in AD. Quantitative analysis using \%VOL identified 28 spots decreased in AD (Fig. 2, Table). One of these spots was identified as GFAP.

\subsection{Protein spots significantly increased in AD brain}

Five protein spots were identified as which are significantly increased in the AD brain. The molecular weights of four increased spots $(213,215,221$, 226) were approximately $52 \mathrm{kDa}$ with isoelectric points ranging from $\mathrm{pH} 4.44$ to 5.04. Two of these four spots were identified as GFAP (Figs. 2 and 3). One low molecular weight protein (about 3 to $10 \mathrm{kDa}$ ) was detected as an increased spot in AD (Fig. 2, Table 1).

\subsection{Protein spots detected only in AD brain}

We detected seven spots present in AD brains but absent in the controls. These spots were small in volume and faint in staining except for the spots designated A107 and A695 (Fig. 2, Table 1).

Analysis with 2-DE has been applied mainly to the proteins from cultured cells, blood components, serum, or body fluids, which are relatively homogeneous [20, $35,36]$. Several improvements have allowed the application of this method to human brain, a heterogeneous tissue containing many cellular components such as a variety of neurons and glia as well as microvessels. Improvement in the resolution and reproducibility of gel images was achieved by using IPG for the first di- mension of electrophoresis [31,37]. Other factors such as autopsy delay, conditions of sonication, and thawing in equilibration solution can influence the results as well. Therefore, an analysis system should allow the extraction of data from a reliable standard spot in often-variable 2-DE images; this was achieved using Melanie II as the data analysis program $[25,38]$. In the present study, reference gels were created by automatically merging a set of gel images that contained at least three pairwise-matched gels.

Application of 2-DE analysis in $\mathrm{AD}$ research has focused mainly on the detection of genetic mutations or post-translational modification of proteins such as tau [2], APP [39], actin [40] and heat shock protein [41]. In a study investigating changes in the expression of brain proteins on 2-DE, Mattila et al. [30] applied IPG to the first dimension of electrophoresis and observed four protein spots in Alzheimer 2-DE which were different in the controls; one spot was undetectable, two spots were significantly weaker and one spot was stronger than those in the controls. Our present results included several more spots which were significantly changed in $\mathrm{AD}$, most likely because we used a narrow $\mathrm{pH}$ range ( $\mathrm{pH} 4$ to 7$)$ and a large (180 $\times 180 \mathrm{~mm}$ ) second-dimension SDS gel, which results in better separation. However, we encountered several unresolved problems in the separation of proteins. We could not demonstrate proteins with a molecular weight greatly exceeding $100 \mathrm{kDa}$, which might be due to the limited ability of heavy proteins to enter the first dimension IPG gel. Studies with larger numbers of cases and investigating more acidic and basic proteins will also be necessary.

\section{Separation of hydrophobic proteins as in the case of membrane proteins}

While IPGs have considerably improved IEF as described above, there are still some drawbacks. It was noted quite quickly that the resolution of some hydrophobic proteins (membrane proteins) was poor and others were lost when separated by IPGs. It is thought that this is because of hydrophobic interactions between the proteins and the basic acrylamide derivatives of the IPG matrix [42]. More recently the protein patterns of some membrane preparations were compared on CAIEF or IPG-IEF 2-D [43]. These experiments clearly showed that the abundance of some proteins in the second dimension was significantly diminished when IPGs were used. Conversely, a wider study by Wilkins et 


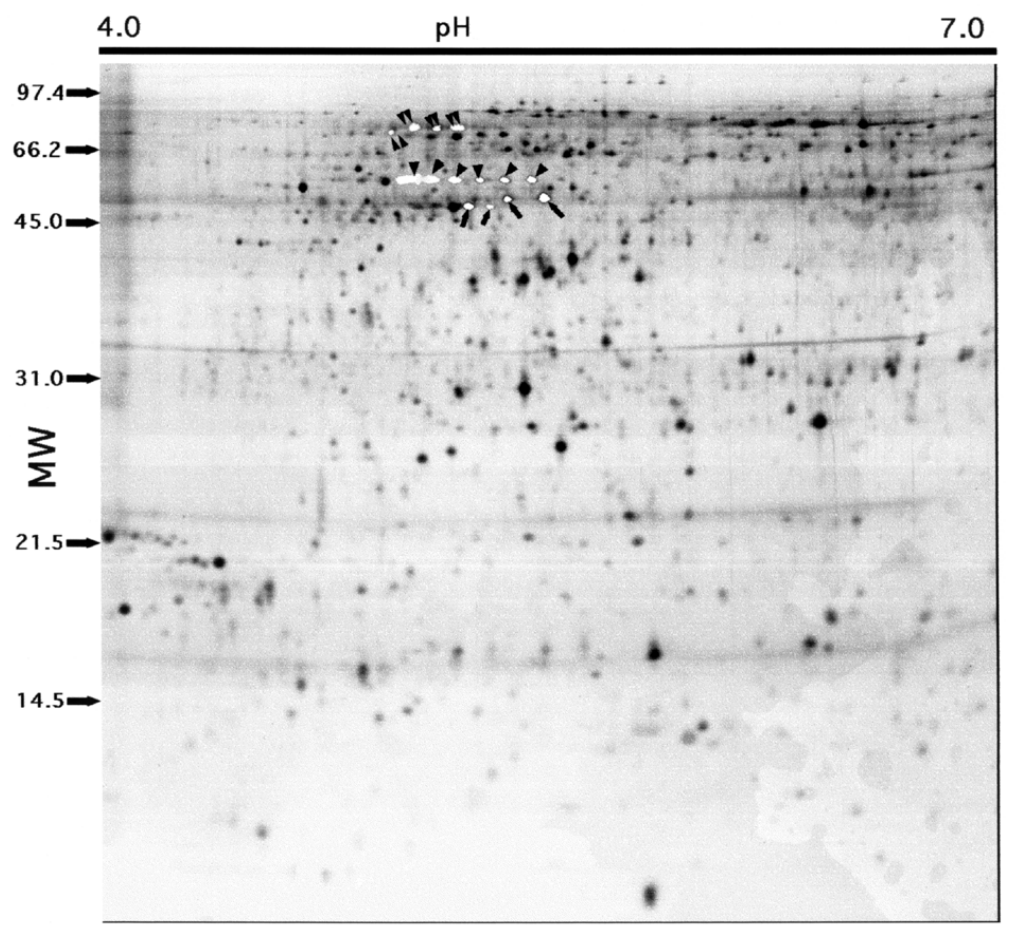

Fig. 1. Typical silver-stained image of 2-DE gels in control human brain. The $\mathrm{pH}$ range is 4 to 7 and the molecular weight markers represent, from top to bottom, 97.4, 66.2, 45.0,31.0, 21.5, and $14.5(\mathrm{kDa})$. This image was obtained through digitization with ARCUS II (Agfa-Gevaert, Mortsel, Belgium), subsequently processed using the Melanie II program. Without processing of this image, such as subtraction of background staining, the spots are detected as well-separated areas throughout the gel. By immunoblotting, two groups of spots are identified on the image as $\beta$-actin (single arrow), GFAP (arrow-heads) and m-calpain (double arrow-heads).

al. [18] indicated that poor protein solubility could be a contributing factor accounting for the absence of hydrophobic proteins on 2-D gels. These authors examined proteins identified on 2-D gels and matched them to the proteins predicted by genome sequencing. Proteins whose overall amino acid composition displayed a hydrophobic bias were almost completely absent from the 2-D gels. Interestingly, the mode of IEF (CA or IPG) did not appear to affect this result, suggesting that the initial sample solubilization was the primary point where hydrophobic proteins were lost.

In recent years, a greater understanding of the biological and pharmacological importance of membranes has prompted significant efforts to improve the separation of these less soluble proteins using the highresolution method of 2-DE. The solubilization and separation of membrane proteins has proved more complex than that of soluble proteins, especially in IPGs for IEF because of their chemical character and membrane compartmentalization. However, with the benefits of IPGs outweighing most drawbacks, improved techniques and strategies for membrane protein separation have slowly evolved over the past two decades.
Reviews reflect upon the problem encountered with 2$\mathrm{DE}$ of membrane proteins and discuss the most recent advances that have been made to overcome the drawbacks identified above [22,44].

\subsection{Protein identification with mass spectrometry}

Since the introduction of MS in protein chemistry, peptide mass fingerprint (PMF) analysis has become the method of choice in high-throughput protein identification. In this approach, the protein of interest, which, in many cases, is purified by 2-D gel electrophoresis, is either enzymatically or chemically cleaved and an aliquot of the peptide mixture thus obtained is analyzed by mass spectrometric techniques. The resulting peptide mass fingerprint is subsequently compared with "virtual" fingerprints obtained by theoretical cleavage of the protein sequences stored in databases and the top-scoring proteins are retrieved as possible candidate proteins. In comparison to other ionization techniques such as Electrospray Ionization (ESI), matrix-assisted laser desorption ionization (MALDI) tolerates moderate buffer and salt concentration in the analyte mix- 


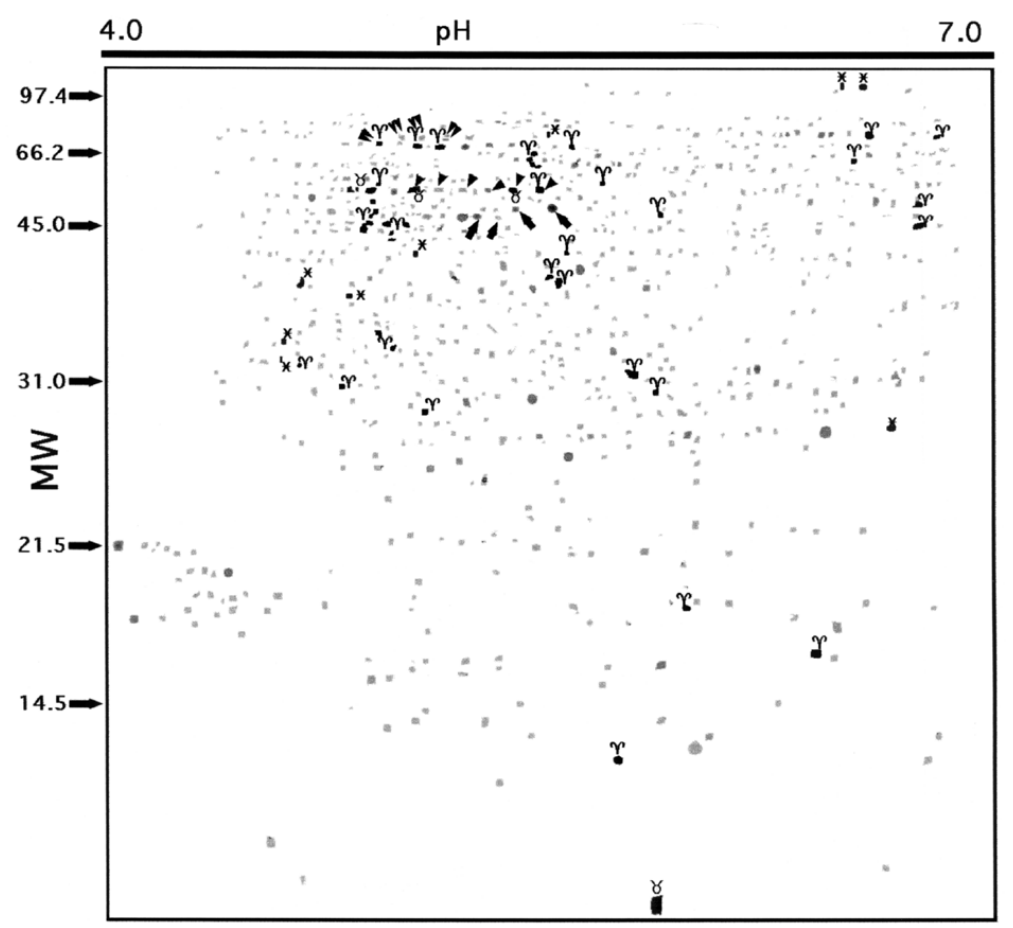

Fig. 2. Profile of spots significantly changed in AD compared with the synthetic reference gel. Gray spots represent proteins unchanged in AD brain. Black spots identified by $\gamma$ represent significantly increased $(p<0.05)$ proteins in AD brain, black spots identified by $\checkmark$ represent

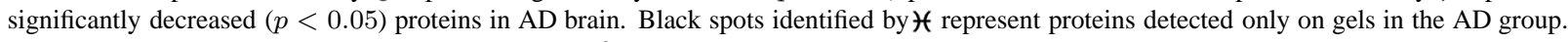
Two groups of spots are identified on the image as $\beta$-actin (single arrow), GFAP (arrow-heads) and m-calpain (double arrow-heads). Two increased spots and one decreased spot were identified as GFAP.

ture and produces almost exclusively singly charged ions [8]. For these reasons it has become the preferred ionization technique for PMF analysis. In the early 90s several research groups reported the use of MALDIPMF in sensitive protein identification [45-47]. It was shown that only a small amount of accurately measured peptide masses is required for unambiguous protein identification and that low pmole to high fmol amounts of gel-separated proteins could be identified using this technique.

ESI mass spectrometry has been particularly successful in the structural and conformational characterization of peptides and proteins [48-50]. Electrospray sources are usually trap mass analyzers, and these instruments have been utilized in protein characterization for years. Recently, a new type of hybrid mass spectrometer has been developed, which combines a quadrupole (Q) analyzer and a time-of-flight (TOF) analyzer in one instrument [51]. In these instruments the quadrupole acts as either an ion guide (MS mode) or as an ion filter (MS/MS mode), whilst the TOF analyzer is responsible for measuring the actual mass of the sample ions. In MS mode the quadrupole simply acts as an ion guide transmitting all ions to the TOF analyzer, and in MS/MS mode it works as an ion filter, allowing only those ions in a predefined mass range to pass through. After passing through the quadrupole analyzer, the filtered ions can be fragmented by collision-induced dissociation in hybrid instruments equipped with a gas collision cell. These daughter ions are then transmitted to the TOF analyzer, where their masses are measured. Using this design it is possible to simultaneously achieve a high resolution and a high sensitivity, due to the very small energy spread and the high transmission of the sample ions [52]. These characteristics give hybrid mass analyzers the capacity to produce high quality MS and MS/Ms spectra from a mol sample quantities [53,54]. Compared to MALDI-MS techniques, sample preparation for ESI-MS is more complex and therefore less well-adapted for high-throughput proteomics. Hybrid Q-TOF mass spectrometers, however, still have a brilliant future and may take a strategic position in the analysis of the site and nature of posttranslational modifications of different proteins. In addition, they remain crucial in the de novo sequencing 
Table 1

List of 2-dimensional electrophoresis protein spots whose density is changed in the AD temporal cortex compared with control temporal cortex from patients with nonneurological disorders

\begin{tabular}{|c|c|c|c|c|c|c|c|}
\hline ID & $\mathrm{pI}$ & MW & $\begin{array}{c}\text { mean OD ratio } \\
\text { (AD/control) }\end{array}$ & ID & $\mathrm{pI}$ & MW & $\begin{array}{c}\text { mean OD ratio } \\
(\mathrm{AD} / \text { control })\end{array}$ \\
\hline \multicolumn{4}{|c|}{ PI/MW of spots decreased in AD brain } & \multicolumn{4}{|c|}{ PI/MW of spots decreased in AD brain (cont.) } \\
\hline 317 & 4.49 & 45 & 0.152 & 74 & 6.80 & 65 & 0.124 \\
\hline 335 & 4.49 & 45 & 0.752 & 536 & 4.29 & 29 & 0.843 \\
\hline 306 & 4.52 & 46 & 0.656 & 572 & 4.41 & 29 & 0.195 \\
\hline 297 & 4.52 & 47 & 0.666 & 505 & 4.60 & 31 & 0.229 \\
\hline 222 & 4.52 & 52 & 0.155 & 606 & 4.72 & 29 & 0.237 \\
\hline 280 & 4.54 & 48 & 0.701 & 759 & 5.43 & 6 & 0.152 \\
\hline 486 & 4.55 & 32 & 0.197 & 545 & 5.49 & 29 & 0.160 \\
\hline 209 & 4.55 & 53 & 0.465 & 581 & 5.57 & 29 & 0.672 \\
\hline 81 & 4.55 & 63 & 0.216 & 716 & 5.68 & 12 & 0.283 \\
\hline 307 & 4.58 & 46 & 0.887 & 732 & 6.42 & 10 & 0.236 \\
\hline 357 & 4.60 & 43 & 0.854 & & & & \\
\hline 347 & 4.60 & 44 & 0.785 & \multicolumn{4}{|c|}{ PI/MW of spots increased in $A D$ brain } \\
\hline 312 & 4.65 & 46 & 0.795 & 215 & 4.44 & 52 & 1.139 \\
\hline 90 & 4.69 & 63 & 0.216 & 221 & 4.47 & 52 & 1.381 \\
\hline 95 & 4.77 & 62 & 0.157 & 213 & 4.68 & 52 & 1.294 \\
\hline 131 & 5.10 & 59 & 0.610 & 226 & 5.04 & 52 & 1.455 \\
\hline 148 & 5.12 & 58 & 0.561 & 765 & 5.57 & 3 & 1.881 \\
\hline 113 & 5.12 & 61 & 0.108 & & & & \\
\hline 217 & 5.14 & 52 & 0.899 & \multicolumn{4}{|c|}{ PI/MW of spots detected only in AD brain } \\
\hline 420 & 5.18 & 38 & 0.163 & A877 & 4.27 & 31 & \\
\hline 424 & 5.21 & 38 & 0.229 & A830 & 4.27 & 32 & \\
\hline 379 & 5.24 & 42 & 0.152 & A695 & 4.29 & 38 & \\
\hline 97 & 5.26 & 62 & 0.199 & A737 & 4.44 & 36 & \\
\hline 201 & 5.38 & 53 & 0.172 & A638 & 4.69 & 42 & \\
\hline 285 & 5.59 & 47 & 0.190 & A107 & 5.18 & 66 & \\
\hline 60 & 6.36 & 66 & 0.962 & A10 & 6.38 & 98 & \\
\hline 137 & 6.41 & 59 & 0.138 & A1023 & 6.44 & 28 & \\
\hline 314 & 6.68 & 46 & 0.176 & A5 & 6.44 & 98 & \\
\hline 260 & 6.68 & 49 & 0.144 & & & & \\
\hline
\end{tabular}

The list includes the spot identification number (ID), isoelectric point (pI) and molecular weight (MW). Quantification of spots was carried out by \% VOL (see Materials and methods). The figure in the "OD ratio (AD/control)" column is the ratio of the mean value of optical density in AD vs control.

of proteins albeit at very low sensitivities which have not yet found their counterparts in databases.

\section{Future direction}

\subsection{High throughput detection system for post-translational modification}

Mass spectrometry, in combination with advanced bioinformatics, has now made it possible to identify proteins present in very small amounts on a 2-D gel with accuracy comparable to traditional Edman sequencing $[45-47,55]$. Hence, with the exponentially growing protein sequence databases, it has become possible to identify the majority of proteins visible on a 2-D gel. The next challenge in proteome analysis is the characterization of post-translational modifications directly from a spot on a gel. In higher eukaryotes a large proportion of proteins present in a given cell are modified and these modifications are often essential for the function of the protein. Modifications can for example, change the solubility or the stability of the protein, act as molecular switches to control the biological activity of the protein or be used to localize the proteins to different compartments in the cell through complex transport pathways. The most common modifications are phosphorylation and glycosylation [56].

Phosphorylation of different amino acids, mainly serine, threonine and tyrosine residues, is of key importance in the regulation of the activity of proteins involved in signal transduction, metabolism or apoptosis in the cell [57]. Therefore, characterization of protein phosphorylation is essential for understanding the true complexity of living cells. It is not surprising that perturbations in the equilibrium of kinase and 
phosphatase activity is fundamentally involved in many diseases such as cystic fibrosis [58], AD [59] and other neurological disorders. Both the quantity and quality of these disease-related phosphorylations should be examined.

\subsection{Protein protein interaction}

Attributing a general role to a gene product based on its homology to other proteins can be a relatively easy (automated) task [60-63]. However, determination of the exact physiological function of a protein often requires a tedious and long-lasting effort, underlining the need for a generic method. Additional knowledge of protein function can be acquired by identifying the physiological binding partners of known function. An important technology is the yeast two-hybrid system [64,65]. However, this approach suffers from the fact that yeast lacks some of the physiology of multicellular organisms, in particular tyrosine kinase. Two-dimensional far-Western immunoblotting is helpful in detecting candidates for binding substrates so as to identify protein-protein interactions.

\section{Conclusion}

2-D PAGE technology and related techniques have reached a new stage in which it is possible to begin to use these methods for clinical applications. While the pathophysiology of the changes found awaits further study, the reference map constructed in the present study is a useful initiation of the human brain proteome database. After establishing the 2-DE database of human brain proteins, we are expanding it by identifying more protein spots, including those specifically changed in AD. We are also interesting to apply the 2DE database to other neurological disorders. We have made these data available on the Internet hoping that all researchers interested in protein analysis of the human brain using 2-DE might take advantage of this 2-DE map as a reference image and collaborate in completing a comprehensive 2-DE database. This will be very helpful in the diagnosis using CSF examination and in the creation of new drug therapy.

\section{Acknowledgments}

We thank Drs. George Perry and Peter J. Whitehouse at Case Western Reserve University (Cleveland, $\mathrm{OH}$ ) for providing us autopsy brains. This work was supported by Grants-in-Aid from the Ministry of Education, Culture, Sports, Science and Technology of Japan, and grants from the Ministry of Health and Welfare of Japan and the Smoking Research Foundation.

\section{References}

[1] C.L. Joachim, J.H. Morris and D.J. Selkoe, Diffuse senile plaques occur commonly in the cerebellum in Alzheimer's disease, Am J Pathol 135 (1989), 309-319.

[2] C. Janke, M. Holzer, J. Klose and T. Arendt, Distribution of isoforms of the microtubule-associated protein tau in grey and white matter areas of human brain: a two-dimensional gelelectrophoretic analysis, FEBS Lett 379 (1996), 222-226.

[3] P. Seubert, T. Oltersdorf and M.G. Lee et al., Secretion of beta-amyloid precursor protein cleaved at the amino terminus of the beta-amyloid peptide, Nature 361 (1993), 260-263.

[4] M. Shoji, T.E. Golde and J. Ghiso et al., Production of the Alzheimer amyloid beta protein by normal proteolytic processing, Science 258 (1992), 126-129.

[5] S.H. Yen, D.W. Dickson, A. Crowe, M. Butler and M.L. Shelanski, Alzheimer's neurofibrillary tangles contain unique epitopes and epitopes in common with the heat-stable microtubule associated proteins tau and MAP2, Am J Pathol 126 (1987), 81-91.

[6] D.H. Cribbs, L.S. Chen, S.M. Bende and F.M. LaFerla, Widespread neuronal expression of the presenilin-1 earlyonset Alzheimer's disease gene in the murine brain, Am J Pathol 148 (1996), 1797-1806.

[7] K. Duff, C. Eckman and C. Zehr et al., Increased amyloidbeta42(43) in brains of mice expressing mutant presenilin 1, Nature 383 (1996), 710-713.

[8] J.B. Fenn, M. Mann, C.K. Meng, S.F. Wong and C.M. Whitehouse, Electrospray ionization for mass spectrometry of large biomolecules, Science 246 (1989), 64-71.

[9] J.E. Taylor, J.R. Tinklenberg and L.F. Eng et al., Association study between Alzheimer's disease and restriction fragment length polymorphisms at the human amyloid beta protein gene locus, Mol Biol Med 5 (1988), 167-172.

[10] H. Tanaka, S. Naruse and K. Seki et al., Absence of linkage disequilibrium at amyloid precursor protein gene locus in Japanese familial Alzheimer's disease with $717 \mathrm{Val} \rightarrow$ Ile mutation, Neurosci Lett 162 (1993), 63-66.

[11] M. Mullan, Familial Alzheimer's disease: second gene locus located [editorial], BMJ 305 (1992), 1108-1109.

[12] T. Tsuji, S. Shimohama, S. Kamiya, T. Sazuka and O. Ohara, Analysis of brain proteins in Alzheimer's disease using highresolution two-dimensional gel electrophoresis, J Neurol Sci 166 (1999), 100-106.

[13] R. Strohman, Epigenesis: the missing beat in biotechnology? Biotechnology (NY) 12 (1994), 156-164.

[14] L. Anderson and J. Seilhamer, A comparison of selected mRNA and protein abundances in human liver, Electrophoresis 18 (1997), 533-537.

[15] P. O'Farrell, High resolution two-dimensional elecrophoresis of proteins, J Biol Chem 250 (1975), 4007-4021.

[16] B. Bjellqvist, J.C. Sanchez and C. Pasquali et al., Micropreparative two-dimensional electrophoresis allowing the separation of samples containing milligram amounts of proteins, Electrophoresis 14 (1993), 1375-1378. 
[17] P.J. Wirth, T.N. Hoang and T. Benjamin, Micropreparative immobilized $\mathrm{pH}$ gradient two-dimensional electrophoresis in combination with protein microsequencing for the analysis of human liver proteins, Electrophoresis 16 (1995), 1946-1960.

[18] M.R. Wilkins, E. Gasteiger, J.C. Sanchez, A. Bairoch and D.F. Hochstrasser, Two-dimensional gel electrophoresis for proteome projects: the effects of protein hydrophobicity and copy number, Electrophoresis 19 (1998), 1501-1505.

[19] A. Gorg, Two-dimensional electrophoresis with immobilized pH gradients: current state, Biochem Soc Trans 21 (1993), 130-132.

[20] J.M. Corbett, M.J. Dunn, A. Posch and A. Gorg, Positional reproducibility of protein spots in two-dimensional polyacrylamide gel electrophoresis using immobilised $\mathrm{pH}$ gradient isoelectric focusing in the first dimension: an interlaboratory comparison, Electrophoresis 15 (1994), 1205-1211.

[21] A. Blomberg, L. Blomberg and J. Norbeck et al., Interlaboratory reproducibility of yeast protein patterns analyzed by immobilized $\mathrm{pH}$ gradient two-dimensional gel electrophoresis, Electrophoresis 16 (1995), 1935-1945.

[22] T. Rabilloud, Solubilization of proteins for electrophoretic analyses, Electrophoresis 17 (1996), 813-829.

[23] T. Tsuji, S. Shimohama, J. Kimura and K. Shimizu, m-Calpain (calcium-activated neutral proteinase) in Alzheimer's disease brains, Neurosci Lett 248 (1998), 109-112.

[24] N.L. Anderson, J. Taylor, A.E. Scandora, B.P. Coulter and N.G. Anderson, The TYCHO system for computer analysis of two-dimensional gel electrophoresis patterns, Clin Chem 27 (1981), 1807-1820.

[25] R.D. Appel, D.F. Hochstrasser and M. Funk et al., The MELANIE project: from a biopsy to automatic protein map interpretation by computer, Electrophoresis 12 (1991), 722735.

[26] P.F. Lemkin, Y. Wu and K. Upton, An efficient disk based data structure for rapid searching of quantitative two-dimensional gel databases, Electrophoresis 14 (1993), 1341-1350.

[27] J.I. Garrels, The QUEST system for quantitative analysis of two-dimensional gels, J Biol Chem 264 (1989), 5269-5282.

[28] P.J. Monardo, T. Boutell, J.I. Garrels and G.I. Latter, A distributed system for two-dimensional gel analysis, Comput Appl Biosci 10 (1994), 137-143.

[29] J.E. Celis, H.H. Rasmussen and P. Gromov et al., The human keratinocyte two-dimensional gel protein database (update 1995): mapping components of signal transduction pathways, Electrophoresis 16 (1995), 2177-2240.

[30] B. Bjellqvist, K. Ek and P.G. Gighetti et al., Isoelectric focusing in immobilized $\mathrm{pH}$ gradients: principle, methodology and some applications, J Biochem Biophys Methods 6 (1982), 317-339.

[31] A. Gorg, W. Postel and S. Gunther et al., Approach to stationary two-dimensional pattern: influence of focusing time and immobiline/carrier ampholytes concentrations, Electrophoresis 9 (1988), 37-46.

[32] D. Cade Treyer, A. Cade, A. Darjo and M. Jouvion Moreno, Isoelectric focusing and titration curves in biomedicine and in agrofood industries: a multimedia teaching program, Electrophoresis 17 (1996), 479-482.

[33] M. Michea-Hamzehpour, J.C. Sanchez and S.F. Epp et al., Two-dimensional polyacrylamide gel electrophoresis isolation and microsequencing of Pseudomonas aeruginosa proteins, Enzyme Protein 47 (1993), 1-8.

[34] A. Gorg, W. Postel, A. Domscheit and S. Gunther, Twodimensional electrophoresis with immobilized $\mathrm{pH}$ gradients of leaf proteins from barley (Hordeum vulgare): method, re- producibility and genetic aspects, Electrophoresis 9 (1988), 681-692.

[35] D.W. Cleveland, S.G. Fischer, M.W. Kirschner and U.K. Laemmli, Peptide mapping by limited proteolysis in sodium dodecyl sulfate and analysis by gel electrophoresis, J Biol Chem. 252 (1977), 1102-1106.

[36] D. Dreher, J.R. Vargas, D.F. Hochstrasser and A.F. Junod, Effects of oxidative stress and $\mathrm{Ca} 2+$ agonists on molecular chaperones in human umbilical vein endothelial cells, Electrophoresis 16 (1995), 1205-1214.

[37] P.K. Sinha, E. Kottgen, M. Stoffler Meilicke, E. Gianazza and P.G. Righetti, Two-dimensional maps in very acidic immobilized pH gradients, J Biochem Biophys Methods 20 (1990), 345-352.

[38] D.F. Hochstrasser, R.D. Appel and R. Vargas et al., A clinical molecular scanner: the Melanie project, MD Comput 8 (1991), 85-91.

[39] L. Autilio Gambetti, A. Morandi and M. Tabaton et al., The amyloid percursor protein of Alzheimer disease is expressed as a $130 \mathrm{kDa}$ polypeptide in various cultured cell types [published erratum appears in FEBS Lett 1989 Feb 27;244:509], FEBS Lett 241 (1988), 94-98.

[40] W. Jabbour, A. Pouplard-Barthelaix, R. Houlgatte and J. Emile, Abnormal expression of actin in lymphocytes of Alzheimer's disease and Down's syndrome patients, $\mathrm{J} \mathrm{Neu}$ roimmunol 38 (1992), 199-208.

[41] N. Perez, J. Sugar and S. Charya et al., Increased synthesis and accumulation of heat shock 70 proteins in Alzheimer's disease, Brain Res Mol Brain Res 11 (1991), 249-254.

[42] H. Kilias, C. Gelfi and P.G. Righetti, Isoenzyme analysis of lichen algae in immobilized $\mathrm{pH}$ gradients, Electrophoresis $\mathbf{9}$ (1988), 187-191.

[43] C. Adessi, C. Miege, C. Albrieux and T. Rabilloud, Twodimensional electrophoresis of membrane proteins: a current challenge for immobilized $\mathrm{pH}$ gradients, Electrophoresis $\mathbf{1 8}$ (1997), 127-135.

[44] P.G. Righetti and E. Gianazza, Isoelectric focusing in immobilized $\mathrm{pH}$ gradients: theory and newer methodology, Methods Biochem Anal 32 (1987), 215-278.

[45] W.J. Henzel, T.M. Billeci and J.T. Stults et al., Identifying proteins from two-dimensional gels by molecular mass searching of peptide fragments in protein sequence databases, Proc Natl Acad Sci USA 90 (1993), 5011-5015.

[46] P. James, M. Quadroni, E. Carafoli and G. Gonnet, Protein identification by mass profile fingerprinting, Biochem Biophys Res Commun 195 (1993), 58-64.

[47] M. Mann, P. Hojrup and P. Roepstorff, Use of mass spectrometric molecular weight information to identify proteins in sequence databases, Biol Mass Spectrom 22 (1993), 338-345.

[48] A. Shevchenko, M. Wilm, O. Vorm and M. Mann, Mass spectrometric sequencing of proteins silver-stained polyacrylamide gels, Anal Chem 68 (1996), 850-858.

[49] M. Wilm, A. Shevchenko and T. Houthaeve et al., Femtomole sequencing of proteins from polyacrylamide gels by nanoelectrospray mass spectrometry, Nature 379 (1996), 466-469.

[50] A. Shevchenko, M. Wilm and M. Mann, Peptide sequencing by mass spectrometry for homology searches and cloning of genes, J Protein Chem 16 (1997), 481-490.

[51] T. Rabilloud, C. Adessi, A. Giraudel and J. Lunardi, Improvement of the solubilization of proteins in two-dimensional electrophoresis with immobilized $\mathrm{pH}$ gradients, Electrophoresis 18 (1997), 307-316.

[52] P. Roepstorff, Mass spectrometry in protein studies from genome to function, Curr Opin Biotechnol 8 (1997), 6-13. 
[53] H.R. Morris, T. Paxton and A. Dell et al., High sensitivity collisionally-activated decomposition tandem mass spectrometry on a novel quadrupole/orthogonal-acceleration time-offlight mass spectrometer, Rapid Commun Mass Spectrom 10 (1996), 889-896.

[54] H.R. Morris, T. Paxton, M. Panico, R. McDowell and A. Dell, A novel geometry mass spectrometer, the Q-TOF, for lowfemtomole/attomole-range biopolymer sequencing, J Protein Chem 16 (1997), 469-479.

[55] M. Mann and M. Wilm, Error-tolerant identification of peptides in sequence databases by peptide sequence tags, Anal Chem 66 (1994), 4390-4399.

[56] R.G. Krishna and F. Wold, Post-translational modification of proteins, Adv Enzymol Relat Areas Mol Biol 67 (1993), 265298.

[57] R. Patarca, Protein phosphorylation and dephosphorylation in physiologic and oncologic processes, Crit Rev Oncog 7 (1996), 343-432.

[58] D.C. Gadsby and A.C. Nairn, Control of CFTR channel gating by phosphorylation and nucleotide hydrolysis, Physiol Rev 79 (1999), S77-S107.

[59] D.P. Hanger, J.C. Betts, T.L. Loviny, W.P. Blackstock and B.H.
Anderton, New phosphorylation sites identified in hyperphosphorylated tau (paired helical filament-tau) from Alzheimer's disease brain using nanoelectrospray mass spectrometry, $J$ Neurochem 71 (1998), 2465-2476.

[60] R. Schaapveld, B. Wieringa and W. Hendriks, Receptor-like protein tyrosine phosphatases: alike and yet so different, $\mathrm{Mol}$ Biol Rep 24 (1997), 247-262.

[61] J.A. Frearson and D.R. Alexander, Protein tyrosine phosphatases in T-cell development, apoptosis and signalling, Immunol Today 17 (1996), 385-391.

[62] H. Sun and N.K. Tonks, The coordinated action of protein tyrosine phosphatases and kinases in cell signaling, Trends Biochem Sci 19 (1994), 480-485.

[63] T.R. Jr. Burke and Z.Y. Zhang, Protein-tyrosine phosphatases: structure, mechanism, and inhibitor discovery, Biopolymers 47 (1998), 225-241.

[64] J. Luban and S.P. Goff, The yeast two-hybrid system for studying protein-protein interactions, Curr Opin Biotechnol 6 (1995), 59-64.

[65] S. Biffo and P.C. Marchisio, The identification of signaling molecules by the yeast two-hybrid system, Methods Mol Biol 96 (1999), 211-220. 


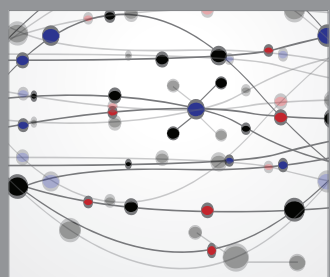

The Scientific World Journal
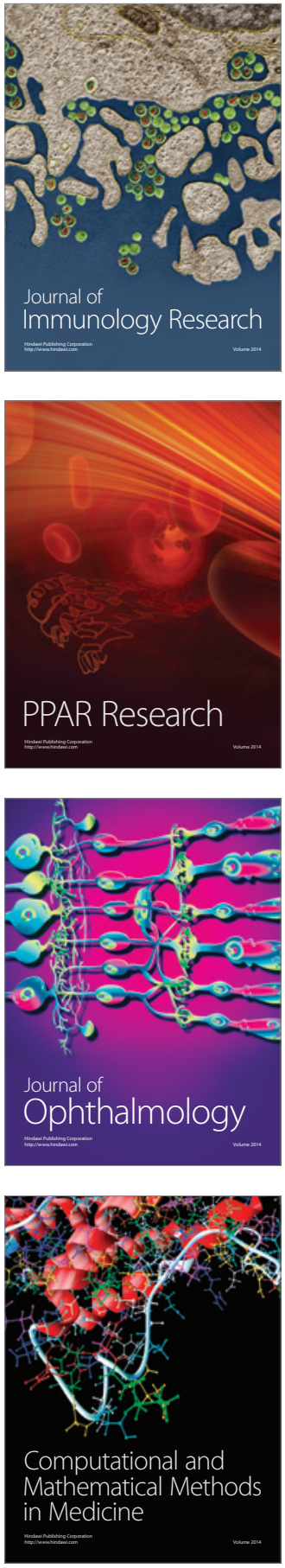

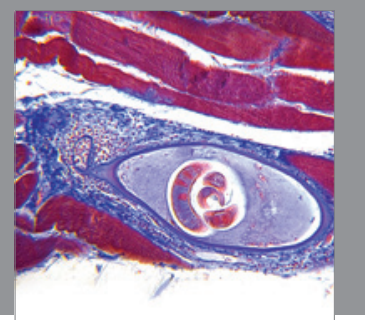

Gastroenterology

Research and Practice
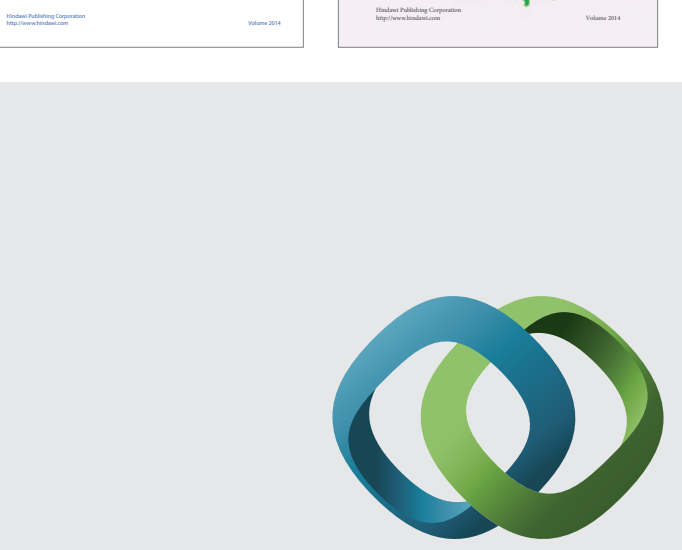

\section{Hindawi}

Submit your manuscripts at

http://www.hindawi.com
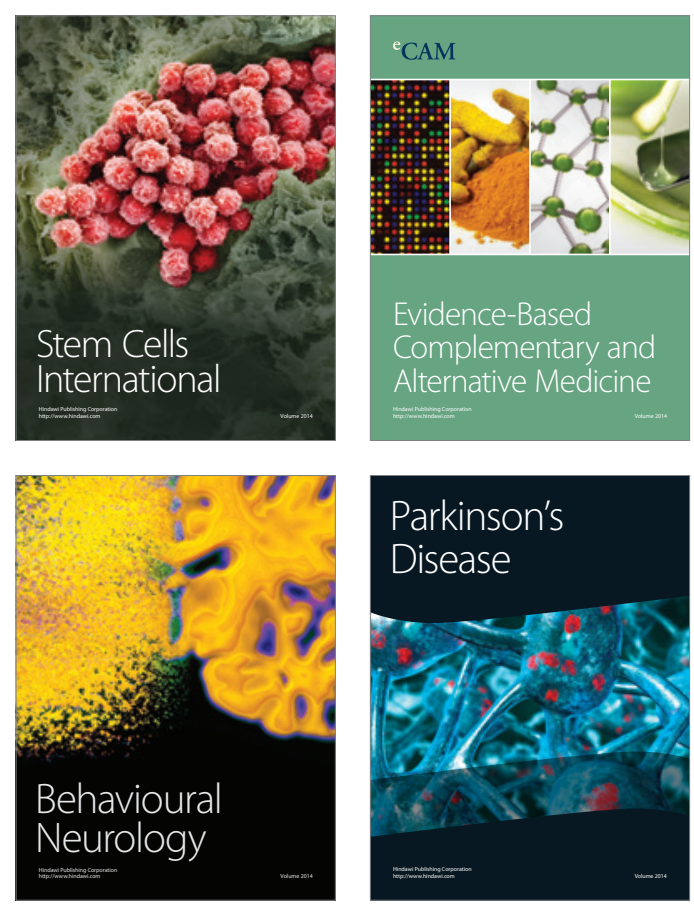

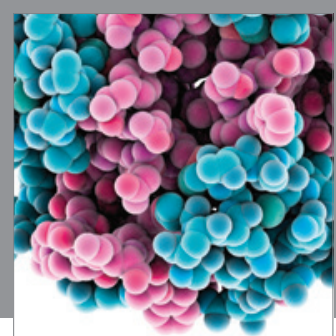

Journal of
Diabetes Research

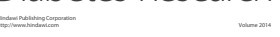

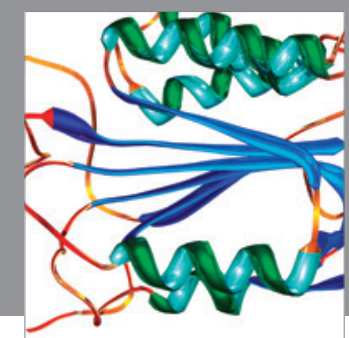

Disease Markers
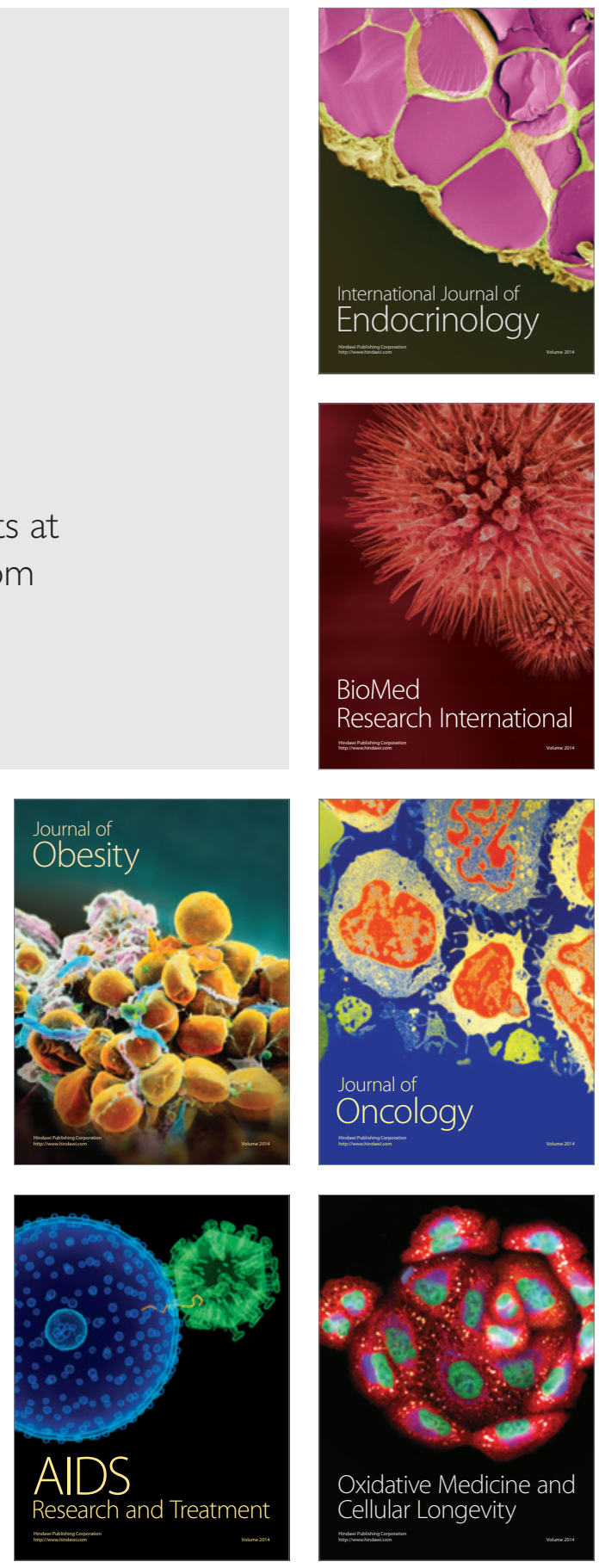